\title{
HYdroxychloroquine to Improve Pregnancy Outcome in Women with AnTIphospholipid Antibodies (HYPATIA) Protocol: A Multinational Randomized Controlled Trial of Hydroxychloroquine versus Placebo in Addition to Standard Treatment in Pregnant Women with Antiphospholipid Syndrome or Antibodies
}

Karen Schreiber, MD, MRCP ${ }^{1,2}$ Karen Breen, MD, MRCPI, FRCPath ${ }^{1}$ Hannah Cohen, MD, FRCP, FRCPath ${ }^{3}$ Soren Jacobsen, MD, DMSc ${ }^{2}$ Saskia Middeldorp, MD, PhD ${ }^{4}$ Sue Pavord, FRCP, FRCPath ${ }^{5}$ Lesley Regan, MD, DSc, FRCOG, FACOG ${ }^{6}$ Dario Roccatello, MD ${ }^{7}$ Susan E. Robinson, MD, FRCP, FRCPath ${ }^{1}$ Savino Sciascia, MD, PhD ${ }^{7}$ Paul T. Seed ${ }^{8}$ Linda Watkins, MRCOG ${ }^{9}$ Beverley J. Hunt, MD, FRCP, FRCPath ${ }^{1}$

1 Thrombosis and Haemophilia Centre, Guy's and St Thomas' NHS Foundation Trust, London, United Kingdom

${ }^{2}$ Copenhagen Lupus and Vasculitis Clinic, Center for Rheumatology and Spine Diseases, Copenhagen University Hospital, Rigshospitalet, Denmark

${ }^{3}$ Haemostasis Research Unit, Department of Haematology, University College London, London, United Kingdom

${ }^{4}$ Department of Vascular Medicine, Academic Medical Center, Amsterdam, The Netherlands.

${ }^{5}$ Department of Haematology, Oxford University Hospitals NHS Foundation Trust, Oxford United Kingdom

6 Obstetrics and Gynaecology Department, St Mary's Hospital, Imperial College, Praed Street, London, United Kingdom

\begin{abstract}
${ }^{7}$ Department of Clinical and Biological Sciences, Center of Research of Immunopathology and Rare Diseases- Coordinating Center of the Network for Rare Diseases of Piedmont and Aosta Valley, San Giovanni Hospital and University of Turin, Torino, Italy

${ }^{8}$ King's Health Partners, King's College London, London, United Kingdom ${ }^{9}$ Liverpool Women's NHS Foundation Trust, Crown Street, Liverpool, United Kingdom
\end{abstract}

Address for correspondence Beverley J. Hunt, MD, FRCP, FRCPath, Thrombosis and Haemophilia Centre, Guy's and St Thomas' NHS Foundation Trust, London, United Kingdom (e-mail: beverley.hunt@gstt.nhs.uk).

Semin Thromb Hemost 2017;43:562-571.

\begin{abstract}
Keywords

- antiphospholipid antibodies

- antiphospholipid syndrome

- obstetric antiphospholipid syndrome

- pregnancy

- hydroxychloroquine

Women with antiphospholipid antibodies (aPL) are at risk of adverse pregnancy outcomes, including recurrent first-trimester pregnancy loss and late pregnancy complications such as preeclampsia, HELLP (hemolysis, elevated liver enzyme levels, and low platelet levels) syndrome, premature delivery, intrauterine growth restriction, placental abruption, and intrauterine death. Current standard care in obstetric antiphospholipid syndrome includes aspirin and heparin and has resulted in live-birth rates of approximately $70 \%$. However, $30 \%$ continue to have pregnancy complications. Hydroxychloroquine (HCQ) is suggested as a new treatment approach, but no randomized controlled trials (RCTs) have assessed its efficacy. This study aims to assess pregnancy outcome in women with aPL treated with HCQ versus placebo in addition to standard treatment. The HYdroxychloroquine to improve Pregnancy outcome in women with AnTIphospholipid Antibodies (HYPATIA) study is a phase IV multicenter RCT, in which pregnant women with persistent aPL will receive either HCQ or placebo in addition to their usual medication. The primary endpoint is a composite of aPL-related adverse pregnancy outcomes: one or more pregnancy loss(es) (either $<10$ or $>10$ weeks of gestation) and premature birth before 34 weeks due to any of the following preeclampsia, eclampsia, or recognized features of placental insufficiency. The HYPATIA study is expected to provide evidence on the effect of HCQ in pregnant women with persistent aPL.
\end{abstract}

Issue Theme Editorial Compilation IV; Guest Editors: Emmanuel J. Favaloro, PhD, FFSc (RCPA), and Giuseppe Lippi, MD.
Copyright @ 2017 by Thieme Medical Publishers, Inc., 333 Seventh Avenue, New York, NY 10001, USA. Tel: +1(212) 584-4662.
DOI https://doi.org/ $10.1055 / \mathrm{s}-0037-1603359$. ISSN 0094-6176. 
Antiphospholipid syndrome (APS) is characterized by the presence of persistent antiphospholipid antibodies (aPL) and clinical manifestations such as thrombosis, microvascular events, and/or pregnancy morbidity and mortality. ${ }^{1}$ The obstetric morbidity in APS includes recurrent pregnancy first-trimester loss, and aPL-related ischemic placental dysfunction such as preeclampsia, eclampsia, HELLP syndrome (hemolysis, elevated liver enzyme levels, and low platelet levels), premature delivery, intrauterine growth restriction, along with intrauterine death. ${ }^{1}$ The aPL included in the classification criteria include lupus anticoagulant, immunoglobulin (Ig)G and IgM anticardiolipin antibodies (aCL), as well as IgG and IgM anti- $\beta_{2}$ glycoproteins I. ${ }^{1}$

The use of low-dose aspirin and low-molecular-weight heparin (LMWH) is the current standard of prevention of aPL-related obstetric complication in women with aPL, and despite a limited body of evidence supporting this treatment, approximately $70 \%$ of women deliver a live, viable infant. ${ }^{2-4}$ However, $30 \%$ of women with aPL continue to have aPLrelated pregnancy complications.

The prevention of obstetric complications of aPL, therefore, remains suboptimal, and other treatment options have been explored. The addition of first-trimester low-dose prednisolone to conventional treatment improved the rate of live births in refractory aPL-related pregnancy loss(es) up to $61 \%{ }^{5}$ Other treatments, such as intravenous Ig, have failed to show benefit., 6 Hydroxychloroquine (HCQ) in APS is receiving increasing international attention, and a group of experts recommended HCQ in addition to standard treatment in those with APS and with previous pregnancy failure on current treatment. ${ }^{8}$ Furthermore, an international task force highlighted the need for clinical trials of HCQ in pregnant women with aPL and APS. ${ }^{9}$ Here, we will review the available basic science and clinical evidence for using HCQ in the setting of aPL. Moreover, we will present the protocol for the first randomized controlled trial (RCT) of HCQ versus placebo in pregnant women with persistent aPL.

\section{What Is Known about HCQ's Action in the Presence of aPL: Basic Science}

Chloroquine and HCQ are weakly basic 4-aminoquinoline compounds. HCQ is the synthetic form of chloroquine and differs only by a hydroxyl group attached to a side chain resulting in a preserved efficacy with a less toxic side-effect profile. ${ }^{10}$ The exact pharmacodynamics and action of HCQ in vivo remain to be fully uncovered, but most likely multiple molecular pathways are involved. HCQ's detailed effects in systemic lupus erythematosus (SLE) have been described elsewhere. ${ }^{11}$ Wallace et al have described HCQ's ability to antagonize Toll-like receptor-mediated immune response activation as one of the most important features in SLE. ${ }^{11}$ It remains unknown which effects of HCQ are important in the immunomodulation of autoimmune diseases and also if different effects are required in different autoimmune diseases. $^{12}$

In retrospective clinical studies, HCQ was associated with a reduction in the risk of thrombosis in lupus patients and is discussed below. The mechanism by which HCQ might have an antithrombotic effect has been studied in a limited way. In vitro studies have shown that HCQ inhibits platelet aggregation and the release of arachidonic acid from aPL-induced stimulated platelets. ${ }^{13}$ Furthermore, Rand et al showed that aPL could disrupt the physiological anticoagulant shield of annexin A5 (AnxA5), leading to exposure of procoagulant phosphatidylserine and subsequently triggering thrombosis. As a novel finding, the group could show that HCQ restores the disruption of natural anticoagulant AnxA5 in patients with aPL. ${ }^{14}$

Tissue factor (TF) is the key initiator of in vivo coagulation and has been implicated in in the pathogenesis of APS. ${ }^{15}$ The ability of aPL to induce TF expression was demonstrated in vitro studies using serum samples from patients with purified aPL, which were added to cells, showing upregulation of TF on monocytes, ${ }^{16,17}$ neutrophils, ${ }^{18}$ and endothelial cells. ${ }^{19}$ Several investigators have found that serum, plasma, purified total IgG, antiB2GPI from APS patients increases TF expression and procoagulant activity on monocytes. ${ }^{20-22}$ LópezPedrera et al showed that aCL IgG stimulates TF expression on circulating monocytes, acting through intracellular pathways including NF-kappaB and MAP kinases. ${ }^{17}$ In mice with aPL-induced fetal loss, neutrophils, in turn, can express TF through aPL-induced complement activation. ${ }^{23}$

We have recently assessed the effect of using HCQ in patients with aPL and APS on plasma biomarkers including soluble TF, which has been shown to be increased in patients with APS. (K.S., K.B., B.J.H., and Ms Kiran Parmar, Prof Jacob Rand, MD, Dr. Wu, MD. The effect of treatment with hydroxychloroquine on biomarkers of hemostasis, complement, inflammation and angiogenesis in patients with antiphospholipid antibodies and antiphosphlipid syndrome. Unpublished Data, 2017). We showed that the use of HCQ was effective to decrease soluble TF levels in patients with aPL and APS 12 weeks after the commencement of HCQ compared with baseline. This may be a mechanism contributing HCQ's antithrombotic effect.

Results from experiments in a murine model by Edwards et al show that HCQ can reverse aPL-induced thrombosis. ${ }^{24}$ The group observed that aPL-injected mice treated with HCQ had a significantly reduced thrombus size, and that thrombus duration was reduced compared with mice treated with placebo. ${ }^{24}$ In vitro studies showed that aPL are directly pathogenic toward trophoblast cells, which is a mechanism cited as a possible cause of recurrent first trimester pregnancy $\operatorname{loss}(\mathrm{es}) .^{25}$ In vitro studies have shown that HCQ reversed the aPL-induced inhibition of the chemokine interleukin 6 (IL-6). ${ }^{26}$ IL-6 released by first trimester trophoblast cells had been shown to drive trophoblast migration (a vital process for implantation) in previous studies by the same group. $^{27}$

We and others have hypothesized that angiogenic molecules may play a role in the pathogenesis of obstetric APS, in light of their role in other placental dysfunction conditions such as preeclampsia. ${ }^{28,29}$ Elevated level of circulating soluble fms-like tyrosine kinase 1 (sFlt1) is thought to cause the maternal expression of preeclampsia. Levels of sFlt1 and 
proangiogenic factors (such as vascular endothelial growth factor and placental growth factor [PIGF]) have been proposed as predictors of preeclampsia in healthy women. ${ }^{28,29}$ In 492 pregnant women from the prospective multicenter cohort, the PROMISSE cohort (PRedictors of pRegnancy outcome: BioMarkers In antiphospholipid Syndrome and Systemic lupus Erythematosus), Kim et al found that the circulating pro- and anti-angiogenic factors such as sFlt1, PIGF, and soluble endoglin (sEng) measured between week 12 and 15 of gestation were predictive of adverse pregnancy outcomes in women with SLE and aPL. ${ }^{30}$

Currently, available evidence suggests that aPL can affect the maternal side of developing placenta by reacting with endometrial cells in the decidua, by which they induce a proinflammatory phenotype and interfere with the physiological implantation process causing first-trimester loss. ${ }^{31}$ These mechanisms are also thought to play a role in the failure of adequate vascularization of developing placenta, ultimately resulting in complications such as preeclampsia. We wished to explore the possible beneficial effect of HCQ on angiogenic biomarkers, as this has not been explored before.

Some studies support the hypothesis that the complement system is activated and plays a role in the pathogenesis of thrombotic and obstetric APS. ${ }^{23,32-35}$ Our group and others have previously confirmed complement activation in patients with isolated aPL and APS (thrombotic and obstetric APS) compared with healthy controls. ${ }^{36,37}$ Our most recent in vivo results assessing the effect of HCQ on complement activation markers C3-des-Arg and Bb in HCQ naive, non-pregnant aPL and APS patients at baseline and 12 weeks after the commencement of HCQ did not show any significant change in these values.

In mice exposed to complement inhibitors and knockout mice which have complement deficiencies, there is inhibition of fetal loss and growth restriction mediated by aPL, 23,32 and also less aPL-induced thrombus. ${ }^{33,34}$ Bertolaccini et al showed a possible protective effect HCQ of in a model of aPLinduced fetal loss. ${ }^{38}$ In their mouse model, the fetuses died in $50 \%$ of pregnant aPL-injected mice, and the survivors were growth-restricted and with smaller placentas compared with control mice. The administration of HCQ to mice exposed to aPL prevented fetal death, increased placental and fetal weight and decreased placental superoxide production (a marker of oxidative stress). ${ }^{38}$ Also, the group reported HCQ-induced complement inhibition in APS patient serum, specifically looking at the common pathway complement activation product C5a-des-Arg after receiving $6.5 \mathrm{mg} / \mathrm{kg}$ HCQ for 6 months. ${ }^{38}$

At an intracellular level, in vitro studies show that aPLs can induce endosomal NADPH (NOX) in endothelial cells and monocytes. ${ }^{39,40}$ NOX is an enzyme complex involved in proinflammatory signaling pathways. ${ }^{12}$ Müller-Calleja et al recently published first in vitro data suggesting that HCQ significantly reduces the induction of endosomal NOX, which leads to a reduction of downstream gene activation. ${ }^{12}$ This "protective" mechanism of HCQ in preventing monocyte activation was confirmed in mice injected with human aPL. ${ }^{12}$

\section{What Is Known about HCQ's Use in Patients with Systemic Lupus Erythematosus and APS: Clinical Studies}

HCQ is traditionally an antimalarial drug and has since the 1950 s been widely used in the treatment of patients with rheumatoid arthritis or systemic connective tissue diseases such as SLE. HCQ is amongst the four drugs currently licensed for the treatment of SLE (other drugs licensed for SLE include aspirin, corticosteroids, and belimumab). ${ }^{41}$ The efficacy of HCQ in patients with SLE has been well described. A cohort study of 150 SLE patients showed that it improves damage free survival, ${ }^{42}$ whereas data of 518 SLE patients from the observational LUMINA cohort showed that its use is associated with a reduced accrual of new disease damage. ${ }^{43}$ RuizIrastorza performed a prospective observational cohort of 232 patients, showing an improved survival in SLE patients taking HCQ. ${ }^{44}$ The use of HCQ in SLE is also associated with a reduced risk of SLE flares. The Canadian Hydroxychloroquine Study Group reported from their RCT, that discontinuation of HCQ was associated with $\mathrm{a} \times 2.5$ relative risk of a clinical lupus flare and that the risk of severe disease flare was significantly higher in patients who had HCQ discontinued. ${ }^{45}$

The antithrombotic effects of HCQ in patients with SLE were reported in data from the LUMINA cohort. In univariate analysis, including 442 SLE patients with aPL of whom 46 were identified having 51 recorded thrombotic events (over 1,446 visits followed over a mean of 88 months), a protective effect of HCQ use was demonstrated (odds ratio [OR], 0.536). This finding is in line with an observational prospective cohort study of 232 SLE patients, in which Cox regression analysis showed that HCQ use was associated with a reduced risk thrombosis (seven events occurred while patients were taking HCQ, seven further events happened after the patient had stopped HCQ whereas 28 events were reported in patients who had never taken HCQ, so yielding a hazard ratio of 0.28$).{ }^{44}$ Likewise, the antithrombotic effect of HCQ could also be demonstrated in several other cohort, casecontrol, and retrospective studies. ${ }^{11}$ Moreover, the safe use of HCQ in pregnancy and lactation has been extensively documented and systematically reviewed. ${ }^{46-48}$ Most data on the use of HCQ in pregnancy stems from patients with SLE. Treatment of pregnant patients with SLE was first described more than three decades ago, and national and international clinical guidelines now recommend using HCQ in pregnant women with underlying autoimmune diseases requiring immunomodulation. ${ }^{49,50}$

The role of HCQ in APS is currently being investigated in thrombotic and obstetric APS. In a prospective nonrandomized study of 40 patients with primary thrombotic APS without underlying SLE, Schmidt-Tanguy et al studied the effect of $400 \mathrm{mg}$ HCQ in addition to oral anticoagulation with vitamin $\mathrm{K}$ antagonists, target international normalized ratio 2 to 3 . None of the patients receiving HCQ along with standard anticoagulation had recurrent thromboembolic events, whereas $30 \%$ in the control group experienced a recurrent event $(p=0.0086) .{ }^{51}$ Unfortunately, the first RCT assessing the role of HCQ role as primary prophylaxis 
in aPL-positive patients has recently been terminated due to manufacturing shortage. ${ }^{52}$

In retrospective studies of pregnant patients with aPL and or APS, we and others showed that HCQ is a candidate for preventing aPL-related adverse pregnancy outcomes. ${ }^{53,54}$ In a retrospective multicenter cohort consisting of 30 APS patients (and 35 pregnancies), Mekinian et al reported that HCQ was associated with fewer first trimester miscarriages (pregnancy losses decreased from 81 to $19 \%, p<0.05$ ) and improved live birth rates in refractory obstetric APS to $78 \%$ $(p<0.05) .{ }^{53}$ In our retrospective observational cohort study of 96 women with 170 pregnancies, we also showed that HCQ treatment was associated with a higher rate of live births $(67 \%$ in HCQ treated vs. $57 \%$ in untreated patients, $p=0.05$ ) and a lower prevalence of pregnancy morbidity (47 vs. 63\%, $p=0.004$ ). Pregnancy duration was longer in patients receiving HCQ compared with those who did not receive HCQ (median 27.6 weeks, range [6-40] vs. 21.5 weeks, range [6-40]; $p=0.03$ ) and fetal losses beyond 10 weeks of gestation were less frequent in women who were treated with HCQ ( 2 vs. $11 \%, p=0.05$ ). Moreover, ischemic placentalmediated complications (preeclampsia, eclampsia, and fetal growth restriction were less prevalent in HCQ-treated women than in the control group ( 2 vs. $10.9 \%, p=0.05$ ). There was a significantly higher rate of women undergoing spontaneous vaginal labor in HCQ women compared with women without HCQ treatment (37.3 vs. $14.3 \%, p=0.01$ ). The association of HCQ with the absence of aPL-related complications in pregnancy was confirmed in multivariate analysis (OR, 2.2; 95\% confidence interval, $1.2-136.1 ; p=0.04) .{ }^{54}$ However, the design of this retrospective study was suboptimal, as the two groups were heterogeneous, with a higher prevalence of SLE amongst those receiving HCQ. These clinical and animal studies, as well as in vitro and ex vivo data, suggest a potential role of HCQ in reducing pregnancy morbidity and mortality in women with aPL. Given all of the available evidence, we proposed HYPATIA study (HYdroxychloroquine to improve Pregnancy outcome in women with AnTIphospholipid Antibodies) which is a randomized controlled multicenter trial of HCQ versus placebo in women with persistent aPL planning for pregnancy. Our hypothesis is that HCQ may improve aPL-related pregnancy complications. ${ }^{55}$

\section{Methods}

\section{Study Design}

The HYPATIA study is an investigator-initiated multicenter, double-blind RCT of women with persistent aPL planning to conceive. Patients will be randomized to HCQ or an identically looking placebo in addition to their usual medication.

Once a woman is enrolled into the HYPATIA study, she will be attending 3 monthly follow-up visits until she conceives (defined as prepregnancy visits). Patients will be starting to take the investigational medicinal product (IMP) as soon as they are randomized (i.e., before conception). If no pregnancy is achieved within 12 months, she will be excluded from the study. Study visits coincide with the usual routine follow-up visits. On these visits, the IMP will repeatedly be dispensed, and compliance will be assessed. All data will be collected in an electronic case record file.

When a woman conceives, she will contact the study team and attend her first pregnancy visit (first-trimester visit). At this visit, a new trial medication will be dispensed. Followup visits for the HYPATIA study purpose will be scheduled once every trimester (defined as 1st, 2nd, and 3rd-trimester visits). Nevertheless, if the participant required closer monitoring, she will be followed up accordingly as per standard of care. Women will be instructed to stop trial medication on the day of delivery and will be asked to hand in the rest of the trial medication at their postpartum follow-up. The postpartum visit shall be completed 6 weeks postpartum.

\section{Patient Recruitment}

Potentially eligible women will be identified in routine outpatient clinics in our participating sites, which include: Guy's and St Thomas' NHS Foundation Trust, London, United Kingdom; Imperial College Health Care NHS Trust, London, United Kingdom; University College London NHS Foundation Trust, London, United Kingdom; Liverpool Women's Hospital NHS Foundation Trust; Oxford University Hospitals NHS Foundation Trust, Oxford, United Kingdom; Academic Medical Center Amsterdam, the Netherlands; University Hospital Torino, Italy; University Hospital Copenhagen, Denmark. Patients will receive full information about the HYPATIA study, and if they decide to take part, a written informed consent will be obtained. Patients will have as much time as they need to consider their participation. Provided all eligibility criteria are fulfilled, study randomization can be undertaken, and the IMP will be dispensed.

Potential participants will be identified by their physician during routine outpatient visits to the trial sites. The HYPATIA study visit assessment schedule is outlined in -Fig. 1.

\section{Inclusion Criteria}

1. Women with known persistent aPL (i.e., isolated persistent aPL or APS) who are planning pregnancy and consent to participate

\section{Exclusion Criteria}

1. Women who are already pregnant

2. Allergy or adverse event to HCQ. Hypersensitivity to the active substance, 4-aminoquinoline or any of the compounds of the IMP or placebo

3. Current treatment with HCQ

4. Age $<18$ and $>45$ years

5 . Body weight $<45 \mathrm{~kg}$

6. Psoriasis

7. Uncontrolled epilepsy

8. Anti-Ro antibodies

9. Renal replacement therapy

10. Other severe active comorbidities (human immunodeficiency virus, hepatitis B)

11. Porphyria

12. History of retinopathy 


\section{HYPATIA trial study procedures}

\begin{tabular}{|c|c|c|c|c|c|c|c|}
\hline & \multicolumn{3}{|c|}{ Pre-pregnancy } & \multicolumn{3}{|c|}{ Pregnancy } & \multirow{2}{*}{$\begin{array}{c}\begin{array}{c}\text { Post- } \\
\text { partum }\end{array} \\
\begin{array}{c}\text { Post- partum } \\
\text { visitf }\end{array}\end{array}$} \\
\hline & $\begin{array}{c}\text { Screening } \\
\text { visit }\end{array}$ & \begin{tabular}{|c|c|c|c|c|} 
Baseline \\
visit
\end{tabular} & $\begin{array}{l}\text { Pre- } \\
\text { pregnancy } \\
\text { visits \& }\end{array}$ & Trimester & Trimestar & Trimester & \\
\hline Written informed consent & $\mathbf{x}$ & & & & & & \\
\hline Demographics & $\mathbf{x}$ & & & & & & \\
\hline Eligibility assessment: & $\mathbf{x}$ & $\mathbf{x}$ & & & & & \\
\hline Past medical history^ & $x$ & & & & & & \\
\hline Smoking history & $\mathbf{x}$ & & & & $\mathrm{x}$ & & \\
\hline Past pregnancy history\$ & $x$ & & & & & & \\
\hline Current medication & $\mathbf{x}$ & $\mathbf{x}$ & $\mathbf{x}$ & $x$ & $\mathbf{x}$ & $\mathbf{x}$ & $\mathbf{x}$ \\
\hline Height & $x$ & & & & & & \\
\hline Weight & $\mathbf{x}$ & & & & & & \\
\hline Collection of blood results ${ }^{\wedge}$ & $x$ & & & & & & \\
\hline Blood pressure \& Pulse & $x$ & & & & & & \\
\hline Urine dip & $\mathbf{x}$ & & & & & & \\
\hline Randomisation & & $\mathbf{x}$ & & & & & \\
\hline Dispensation IMP & & $x$ & $\mathbf{x}$ & $\mathbf{x}$ & $\mathbf{x}$ & $\mathbf{x}$ & \\
\hline Adverse events monitoring & & $\mathbf{x}$ & $\mathbf{x}$ & $\mathbf{x}$ & $\mathbf{x}$ & $\mathbf{x}$ & $\mathbf{x}$ \\
\hline Info about current pregnancy" & & & & $\bar{x}$ & $\mathbf{x}$ & $x$ & \\
\hline Compliance check€ & & & $\mathbf{x}$ & $x$ & $x$ & $x$ & $\mathbf{x}$ \\
\hline Blood for additional research & & & $x \sqrt{1}$ & xI & $x \|$ & $x \boldsymbol{I}$ & $\mathbf{x}$ \\
\hline Growth scans & & & & & $\mathbf{x}$ & $\mathbf{x}$ & $\mathbf{x}$ \\
\hline Uterine Artery Doppler (20-24 weeks) & & & & & $\mathbf{x t}$ & & \\
\hline Child gender & & & & & & & $\mathbf{x}$ \\
\hline Birth date & & & & & & & $\mathbf{x}$ \\
\hline Child APGAR at $5 \mathrm{~min}$ & & & & & & & $\mathbf{x}$ \\
\hline Days of admission (mother\&child) & & & & & & & $\mathbf{x}$ \\
\hline Gestational age & & & & & & & $\mathbf{x}$ \\
\hline Mode of delivery (Cesarean Y/N) & & & & & & & $\mathbf{x}$ \\
\hline Birth weight & & & & & & & $\mathbf{x}$ \\
\hline $\begin{array}{l}\text { Neonatal morbidity (neonatal } \\
\text { bleeding or thrombotic } \\
\text { complications, infections, congenital } \\
\text { abnormalities, days in neonatal } \\
\text { special care) }\end{array}$ & & & & & & & $\mathbf{x}$ \\
\hline \multicolumn{8}{|c|}{$\begin{array}{l}\text { - These visits can all be completed on the same day. Consent within } 3 \text { months. } \\
\text { Follow up visit should be completed within } 6 \text { weeks. If the patient is unable to attend clinic within the timetrame of } 6 \text { weeks, the study team can } \\
\text { contad the participant to abtain the study information. }\end{array}$} \\
\hline \multicolumn{8}{|c|}{ 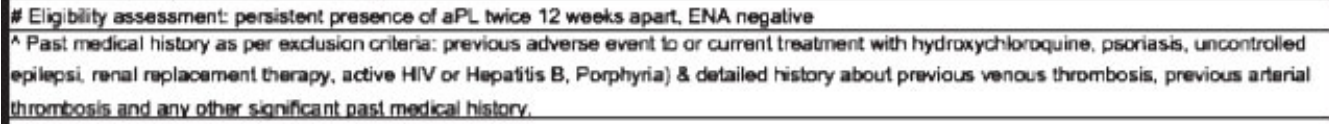 } \\
\hline \multirow{3}{*}{\multicolumn{8}{|c|}{ 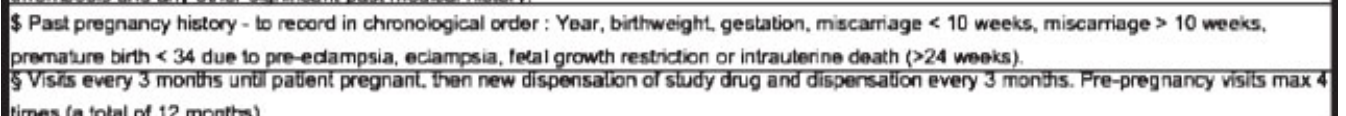 }} \\
\hline & & & & & & & \\
\hline & & & & & & & \\
\hline \multicolumn{8}{|c|}{$\begin{array}{l}\text { times (a total of } 12 \text { montrs) } \\
\text { IIVF pregnancy (YIN), singielontwin or triplet pregnancy, current cestation, estimated dace of delivery }\end{array}$} \\
\hline \multicolumn{8}{|c|}{ E Compliance check (Kit number to be noted, date of current kit stanted \& ended, number of tabiets taken, number of tabiets missed) } \\
\hline \multicolumn{8}{|c|}{ IOnly for patients at SI Thomas' Hospital: optional research bloods. } \\
\hline \multicolumn{8}{|c|}{ 4 Data can be collecied on 2nd or 3rd Trimester vist. All scans should be recorded on eCRF. } \\
\hline 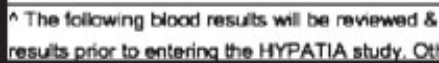 & 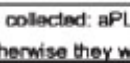 & & & & & & \\
\hline
\end{tabular}

Fig. 1 The HYPATIA study visit assessment schedule. HYPATIA, HYdroxychloroquine to Improve Pregnancy Outcome in Women with AnTIphospholipid Antibodies.

13. History of galactose intolerance, lactase deficiency, or glucose-galactose malabsorption

14. Participation in any other IMP trial at the time of consent 15. Previous pregnancy failure on HCQ
Laboratory assessment for aPL diagnosis: For the purpose of the HYPATIA study aPL positivity is defined by the presence of a positive test for anticardiolipin antibodies (IgG/IgM isotypes $>$ 95th percentile) and/or lupus anticoagulant 


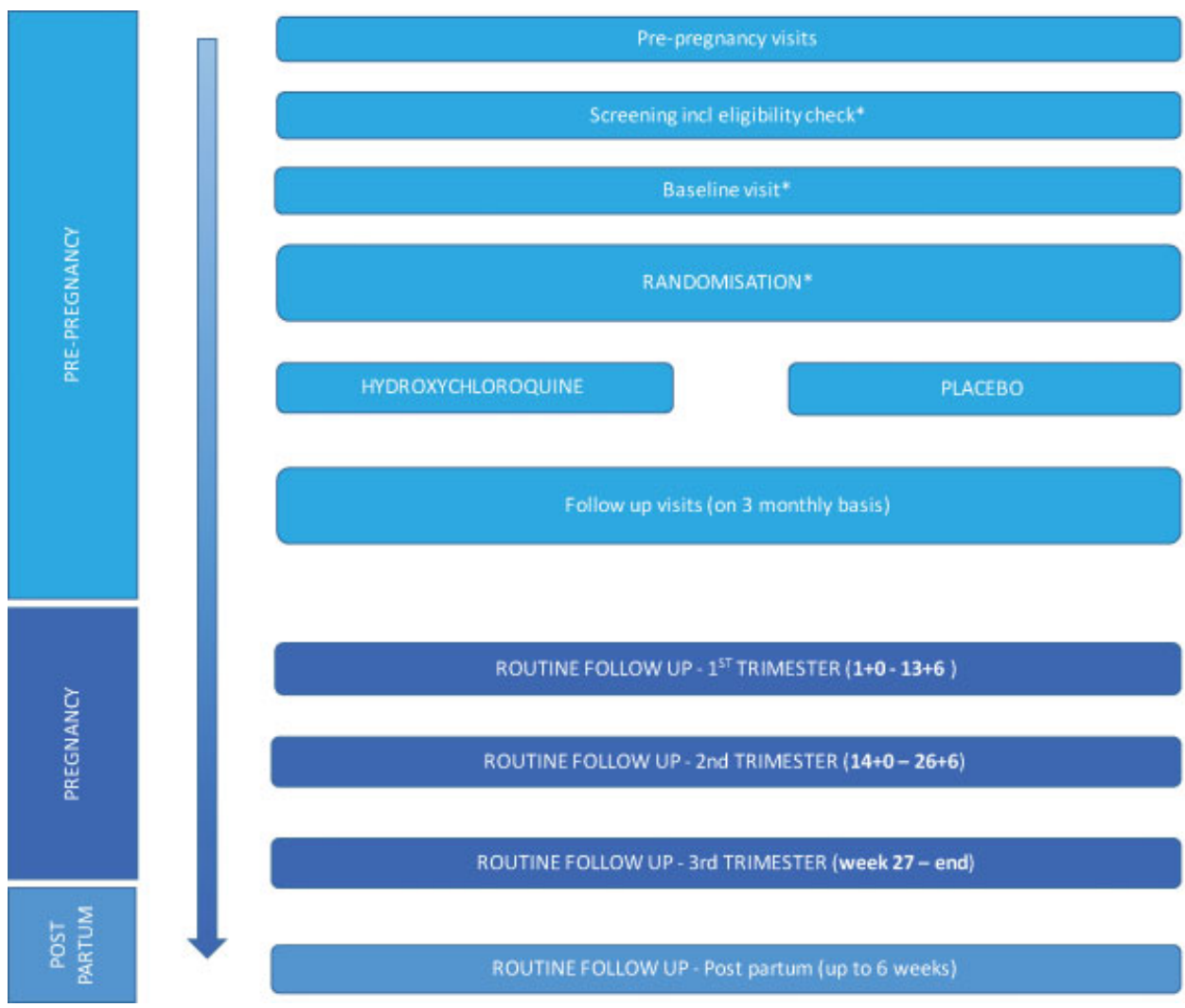

Fig. 2 The HYPATIA study trial flow chart. HYPATIA, HYdroxychloroquine to Improve Pregnancy Outcome in Women with AnTIphospholipid Antibodies.

and/or anti- $\beta_{2}$-glycoprotein-I ( $\operatorname{IgG} / \operatorname{IgM}$ isotypes $>95$ th percentile), on two or more consecutive occasions more than 12 weeks apart. All participating sites will be expected to have established their own 95th percentile, and data on absolute values (for aCL and anti- $\beta_{2}$-glycoprotein-I), lower and upper normal value will be collected in the electronic database. aPL are assessed according to standard established methods. ${ }^{56,57}$

\section{Duration of Trial}

The HYPATIA study will last for a total of 36 months, which includes recruitment and follow-up of patients. The end of the trial is defined as database lock. Each individual will remain in the study as long as their time to conception (up to maximum 12 months), their full length of pregnancy until delivery. In total 328 pregnant women (sample size calculation is detailed below) will be included in the study. The trial flow chart is summarized in - Fig. $\mathbf{2}$.

\section{Primary Outcome Measures}

The primary endpoint is a composite of three principal aPL-related adverse pregnancy outcomes: one or more pregnancy loss(es) (either $<10$ weeks gestation or beyond 10 weeks of gestation of a morphologically normal fetus documented by ultrasound or by direct examination of the fetus) and premature birth of a morphologically normal neonate before 34 weeks due to any of preeclampsia, eclampsia, and recognized features of placental insufficiency.
Premature birth for other reasons will not be included. The components of the primary endpoint will each be presented as secondary endpoints (below).

\section{Secondary Outcomes Measures}

The predefined secondary endpoints include: (1) Pregnancy loss $<10$ weeks gestation, (2) pregnancy loss $>10$ th week of gestation of a morphologically normal fetus documented by ultrasound or by direct examination of the fetus, (3) premature birth of a morphologically normal neonate $<34$ weeks due to any of preeclampsia, eclampsia, recognized features of placental insufficiency, (4) gestational age at delivery, (5) birth weight, (6) cesarean delivery, (7) Apgar score $<7$ at 5 minutes, (8) neonatal morbidity (bleeding or thrombotic complications, infections, congenital abnormalities), (9) days to hospital discharge following delivery (mother and child), (10) thrombotic events in the mother during pregnancy and 6 weeks postpartum, and (11) days of the neonate in special care.

Suspected unexpected serious adverse reactions and other serious adverse events (SAEs) will be reported to the regulatory authorities and the research ethics committees, as appropriate. All SAE reports received from the study sites will be reviewed by independent medically qualified staff.

SAEs that do not require reporting: Pregnancy itself is well known to potentially causing nausea and tiredness due to several physiological adjustments of the body especially in the first trimester. Any symptoms thought to be related to the physiological changes in pregnancy do not require reporting. 
In patients with aPL, we expect pregnancy complications. Therefore, aPL-related pregnancy complications, such as miscarriage $<10$ weeks' gestation, miscarriage $>$ weeks 10 , premature birth $<34$ weeks due to preeclampsia, eclampsia or fetal growth restriction, and stillbirth shall not be reported as SAE. For these events we have designed a special section in the electronic database "adverse pregnancy outcomes," which shall be completed in this case. Any other adverse events will be reported.

\section{Randomization}

Randomization will be performed by an independent individual at King's Clinical Trials Unit (King's CTU), using a computer-based minimization process, adjusting for thrombosis (yes/no), previous adverse pregnancy outcomes (yes/ no), and previous pregnancy (yes/no).

The investigators and trial subjects will remain blinded to treatment. The trial pharmacist will be blinded too. Unique pack numbers for the trial medication will be generated for the active and placebo products and will be sent with randomization emails. The trial statistician will know only A versus B, with the meaning of A and B held by King's CTU.

\section{Statistical Analysis}

\section{Sample Size}

The sample size was calculated based on our audit data. Retrospective data from St Thomas' Hospital showed that treatment with $\mathrm{HCQ}$ was associated with a higher rate of live birth (67\% in women with SLE and aPL treated with HCQ vs. $57 \%$ in women in the control group $p=0.05$ ) and a lower prevalence of aPL-related pregnancy morbidity (47 vs. $63 \%$ $p=0.004){ }^{8}$ The trial is powered to detect a $16 \%$ reduction in pregnancy morbidity, which is the main outcome of the study (total sample size 328 ). A minimum of 328 women will, therefore, be randomized. The calculation was made on the following: significance $\alpha=0.05$, power $1-\beta 80 \%$, and percentage cross $5 \%$.

Primary statistical analysis: Percentages in both arms of the study will be compared using binomial regression with a log link, adjusting for the minimization variables to give risk ratios. Risk differences will be estimated similarly. Continuous measures will be compared using linear regression adjusting for minimization variables and baseline measurement of the outcome (where available). Estimates will be given with $95 \%$ confidence intervals.

Results will be considered significant at the $5 \%$ level. However, attention will be paid to the totality of results and the exact size of the $p$ value. Smaller values will be treated as stronger evidence against the null hypothesis. The intention-to-treat principle will be followed in the main analysis, but a secondary per-protocol analysis will also be performed to provide additional information on the nature of the treatment effect.

Three subgroup analyses are planned: by the previous thrombosis; previous adverse pregnancy outcomes; previous pregnancy. In each case, the primary outcome will be analyzed in each subgroup, and an interaction test per- formed for evidence of a difference in treatment effect between the groups.

No formal interim analyses are planned. The Data Monitoring Committee will aim to meet in person at least every 6 months and will consider the results so far. They are empowered to request that the trial stops if there is overwhelming evidence for or against one treatment, such that to randomize further patients would be unethical.

Competent authority approval in the United Kingdom has passed the initial assessment and ethics approval is currently awaited. As a European multicenter trial, we are following the "voluntary harmonization procedure." 58

\section{Discussion}

We hypothesize that the addition of HCQ to the standard-ofcare treatment in pregnant women with aPL will improve aPL-related adverse pregnancy outcomes. Current data to suggest a role of $\mathrm{HCQ}$ in these patients is inadequate; hence, we have designed an adequately powered RCT. ${ }^{53,54}$

We have shown that with a rigorously applied local protocol around $70 \%$ of pregnant women with aPL/APS will deliver a viable infant. ${ }^{2}$ The current management includes LMWH and/or low-dose aspirin. As mentioned above, this does not prevent all maternal, fetal, and neonatal complications. Our retrospective study suggests that HCQ has a beneficial effect in women with aPL, as the treatment with HCQ was associated with a higher rate of live births (67 vs. $57 \%, p=0.05$ ) and a lower prevalence of aPL-related pregnancy morbidity (47 vs. $63 \%, p=0.004$ ). ${ }^{54}$ Despite the heterogeneity in the two groups in terms of SLE prevalence and previous pregnancy history, our results support the concept that women with aPL may benefit from treatment with HCQ during pregnancy to improve pregnancy outcome.

We have also conducted a systematic review of the evidence of HCQ in obstetric APS, which confirmed a lack of evidence. $^{8}$ We, therefore, performed an expert-based clinical judgment consensus, which is an accepted approach to address a specific and clinically relevant question in an area were best clinical practice is uncertain. The experts agreed that HCQ could be considered in selected cases of patients with obstetric APS or after the failure of standard treatment with aspirin and any heparin-based agent. Moreover, the majority of experts considered adding HCQ in specific scenarios, such as women with previous thrombosis or previous ischemic placental medicated complications. ${ }^{8}$

The ideal RCT to answer the question of the benefit of HCQ in pregnancy in those with aPL would be to investigate women with refractory obstetric APS, that is, those who have failed conventional treatment, and then to randomize this group to HCQ versus placebo. However, after reviewing our patient group and discussing with all our trial centers, we calculated that it would take 10 years to recruit adequate women to a study of this design. We have hence chosen to study women with aPL rather than women with refractory obstetric APS because it not only practically feasible but also supported by the results of our previous retrospective study. 
Some may argue that that the inclusion of women with aPL above the 95th percentile as opposed to the defined 99th percentile in the Miyakis criteria is a fault in the study. ${ }^{1}$ However, it is our and others experience, that low-level aPL are highly relevant in the setting of obstetric APS. ${ }^{59,60}$ This is in line with the observation of Ruffatti et al, that women with purely obstetric APS have lower aCL antibody titers compared with patients with thrombotic APS. ${ }^{61}$ Moreover, retrospective data and data from a cohort study suggest that lowtiter aCL, defined as those between the 95th and 99th percentiles, are still of clinical significance for women with obstetric APS. ${ }^{62,63}$

Although this study includes women planning to conceives, and will, therefore, be perceived by some as a difficult study. However, some of our HYPATIA study team members have experience in successfully conducting RCTs in which women were recruited preconceptionally. ${ }^{64}$ We are involving centers that are expert tertiary centers for managing women with aPL and managing them preconception and throughout pregnancy. Recruitment estimates of some patients that will be entered into the study have been obtained from each center and exceed the proposed number by approximately $20 \%$, and we fully expect completion of the study in the allotted time.

HYPATIA is the first trial to randomize HCQ versus placebo in pregnant women with persistent aPL, and our results will provide an evidence-base for the decision to use of HCQ in these patients.

Funding

The HYPATIA study is funded by the NIHR Research for Patient Benefit (RfBP).

\section{Notes}

B.J.H. and K.S. have designed the trial. All authors and collaborators have reviewed and edited the draft version of the article and approved the final version. All authors have approved the final article submitted. Trial registration: EUDRACT: 2016-002256-25.

\section{Acknowledgments}

The investigators are grateful to the patients involved in the trial and to the research staff at all collaborating university hospitals.

\section{References}

1 Miyakis S, Lockshin MD, Atsumi T, et al. International consensus statement on an update of the classification criteria for definite antiphospholipid syndrome (APS). J Thromb Haemost 2006; 4(02):295-306

2 Bramham K, Hunt BJ, Germain S, et al. Pregnancy outcome in different clinical phenotypes of antiphospholipid syndrome. Lupus 2010;19(01):58-64

3 Bouvier S, Cochery-Nouvellon E, Lavigne-Lissalde G, et al. Comparative incidence of pregnancy outcomes in treated obstetric antiphospholipid syndrome: the NOH-APS observational study. Blood 2014;123(03):404-413
4 Mak A, Cheung MW, Cheak AA, Ho RC. Combination of heparin and aspirin is superior to aspirin alone in enhancing live births in patients with recurrent pregnancy loss and positive anti-phospholipid antibodies: a meta-analysis of randomized controlled trials and meta-regression. Rheumatology (Oxford) 2010;49(02): 281-288

5 Bramham K, Thomas M, Nelson-Piercy C, Khamashta M, Hunt BJ. First-trimester low-dose prednisolone in refractory antiphospholipid antibody-related pregnancy loss. Blood 2011;117(25): 6948-6951

6 Branch DW, Peaceman AM, Druzin M, et al; The Pregnancy Loss Study Group. A multicenter, placebo-controlled pilot study of intravenous immune globulin treatment of antiphospholipid syndrome during pregnancy. Am J Obstet Gynecol 2000;182(1 Pt 1): 122-127

7 Triolo G, Ferrante A, Ciccia F, et al. Randomized study of subcutaneous low molecular weight heparin plus aspirin versus intravenous immunoglobulin in the treatment of recurrent fetal loss associated with antiphospholipid antibodies. Arthritis Rheum 2003;48(03):728-731

8 Sciascia S, Branch DW, Levy RA, et al. The efficacy of hydroxychloroquine in altering pregnancy outcome in women with antiphospholipid antibodies. Evidence and clinical judgment. Thromb Haemost 2016;115(02):285-290

9 Erkan D, Aguiar CL, Andrade D, et al. 14th International Congress on Antiphospholipid Antibodies: task force report on antiphospholipid syndrome treatment trends. Autoimmun Rev 2014; 13(06):685-696

10 Wallace DJ. The history of antimalarials. Lupus 1996;5(Suppl 1): S2-S3

11 Wallace DJ, Gudsoorkar VS, Weisman MH, Venuturupalli SR. New insights into mechanisms of therapeutic effects of antimalarial agents in SLE. Nat Rev Rheumatol 2012;8(09):522-533

12 Müller-Calleja N, Manukyan D, Canisius A, Strand D, Lackner KJ. Hydroxychloroquine inhibits proinflammatory signalling pathways by targeting endosomal NADPH oxidase. Ann Rheum Dis 2016; annrheumdis-2016-210012

13 Pierangeli SS, Vega-Ostertag M, Harris EN. Intracellular signaling triggered by antiphospholipid antibodies in platelets and endothelial cells: a pathway to targeted therapies. Thromb Res 2004;114(5-6):467-476

14 Rand JH, Wu XX, Quinn AS, et al. Hydroxychloroquine protects the annexin A5 anticoagulant shield from disruption by antiphospholipid antibodies: evidence for a novel effect for an old antimalarial drug. Blood 2010;115(11):2292-2299

15 Giannakopoulos B, Krilis SA. The pathogenesis of the antiphospholipid syndrome. N Engl J Med 2013;368(11):1033-1044

16 Sorice M, Longo A, Capozzi A, et al. Anti-beta2-glycoprotein I antibodies induce monocyte release of tumor necrosis factor alpha and tissue factor by signal transduction pathways involving lipid rafts. Arthritis Rheum 2007;56(08):2687-2697

17 López-Pedrera C, Buendía P, Cuadrado MJ, et al. Antiphospholipid antibodies from patients with the antiphospholipid syndrome induce monocyte tissue factor expression through the simultaneous activation of NF-kappaB/Rel proteins via the p38 mitogenactivated protein kinase pathway, and of the MEK-1/ERK pathway. Arthritis Rheum 2006;54(01):301-311

18 Ritis K, Doumas M, Mastellos D, et al. A novel C5a receptor-tissue factor cross-talk in neutrophils links innate immunity to coagulation pathways. J Immunol 2006;177(07):4794-4802

19 Vega-Ostertag M, Casper K, Swerlick R, Ferrara D, Harris EN, Pierangeli SS. Involvement of p38 MAPK in the up-regulation of tissue factor on endothelial cells by antiphospholipid antibodies. Arthritis Rheum 2005;52(05):1545-1554

20 Wolberg AS, Roubey RA. Mechanisms of autoantibody-induced monocyte tissue factor expression. Thromb Res 2004;114(5-6): 391-396 
21 Kornberg A, Blank M, Kaufman S, Shoenfeld Y. Induction of tissue factor-like activity in monocytes by anti-cardiolipin antibodies. J Immunol 1994;153(03):1328-1332

22 Reverter JC, Tàssies D, Font J, et al. Effects of human monoclonal anticardiolipin antibodies on platelet function and on tissue factor expression on monocytes. Arthritis Rheum 1998;41(08): 1420-1427

23 Girardi G, Berman J, Redecha P, et al. Complement C5a receptors and neutrophils mediate fetal injury in the antiphospholipid syndrome. J Clin Invest 2003;112(11):1644-1654

24 Edwards MH, Pierangeli S, Liu X, Barker JH, Anderson G, Harris EN. Hydroxychloroquine reverses thrombogenic properties of antiphospholipid antibodies in mice. Circulation 1997;96(12): 4380-4384

25 Mulla MJ, Brosens JJ, Chamley LW, et al. Antiphospholipid antibodies induce a pro-inflammatory response in first trimester trophoblast via the TLR4/MyD88 pathway. Am J Reprod Immunol 2009;62(02):96-111

26 Albert CR, Schlesinger WJ, Viall CA, et al. Effect of hydroxychloroquine on antiphospholipid antibody-induced changes in first trimester trophoblast function. Am J Reprod Immunol 2014; 71(02):154-164

27 Mulla MJ, Myrtolli K, Brosens JJ, et al. Antiphospholipid antibodies limit trophoblast migration by reducing IL- 6 production and STAT3 activity. Am J Reprod Immunol 2010;63(05):339-348

28 Levine RJ, Maynard SE, Qian C, et al. Circulating angiogenic factors and the risk of preeclampsia. N Engl J Med 2004;350 (07):672-683

29 Levine RJ, Lam C, Qian C, et al; CPEP Study Group. Soluble endoglin and other circulating antiangiogenic factors in preeclampsia. $\mathrm{N}$ Engl J Med 2006;355(10):992-1005

30 Kim MY, Buyon JP, Guerra MM, et al. Angiogenic factor imbalance early in pregnancy predicts adverse outcomes in patients with lupus and antiphospholipid antibodies: results of the PROMISSE study. Am J Obstet Gynecol 2016;214(01):108.e1-108.e14

31 Pierangeli SS, Chen PP, Raschi E, et al. Antiphospholipid antibodies and the antiphospholipid syndrome: pathogenic mechanisms. Semin Thromb Hemost 2008;34(03):236-250

32 Holers VM, Girardi G, Mo L, et al. Complement C3 activation is required for antiphospholipid antibody-induced fetal loss. J Exp Med 2002;195(02):211-220

33 Pierangeli SS, Girardi G, Vega-Ostertag M, Liu X, Espinola RG, Salmon J. Requirement of activation of complement C3 and C5 for antiphospholipid antibody-mediated thrombophilia. Arthritis Rheum 2005;52(07):2120-2124

34 Fischetti F, Durigutto P, Pellis V, et al. Thrombus formation induced by antibodies to beta2-glycoprotein I is complement dependent and requires a priming factor. Blood 2005;106(07): 2340-2346

35 Meroni PL, Borghi MO, Raschi E, Tedesco F. Pathogenesis of antiphospholipid syndrome: understanding the antibodies. Nat Rev Rheumatol 2011;7(06):330-339

36 Breen KAS, Seed P, Parmar K, Moore GW, Stuart-Smith SE, Hunt BJ. Complement activation in patients with isolated antiphospholipid antibodies or primary antiphospholipid syndrome. Thromb Haemost 2012;107(03):423-429

37 Oku K, Amengual O, Zigon P, Horita T, Yasuda S, Atsumi T. Essential role of the p38 mitogen-activated protein kinase pathway in tissue factor gene expression mediated by the phosphatidylserinedependent antiprothrombin antibody. Rheumatology (Oxford) 2013;52(10):1775-1784

38 Bertolaccini ML, Contento G, Lennen R, et al. Complement inhibition by hydroxychloroquine prevents placental and fetal brain abnormalities in antiphospholipid syndrome. J Autoimmun 2016;75:30-38

39 Prinz N, Clemens N, Canisius A, Lackner KJ. Endosomal NADPHoxidase is critical for induction of the tissue factor gene in monocytes and endothelial cells. Lessons from the antiphospholipid syndrome. Thromb Haemost 2013;109(03):525-531
40 Müller-Calleja N, Köhler A, Siebald B, et al. Cofactor-independent antiphospholipid antibodies activate the NLRP3-inflammasome via endosomal NADPH-oxidase: implications for the antiphospholipid syndrome. Thromb Haemost 2015;113(05):1071-1083

41 U.S. Food and Drug Administration. Lupus therapies continue to evolve. Available at: https://www.fda.gov/forconsumers/consumerupdates/ucm398682.htm. Accessed May 27, 2017

42 Molad Y, Gorshtein A, Wysenbeek AJ, et al. Protective effect of hydroxychloroquine in systemic lupus erythematosus. Prospective long-term study of an Israeli cohort. Lupus 2002;11(06):356-361

43 Fessler BJ, Alarcón GS, McGwin G Jr, et al; LUMINA Study Group. Systemic lupus erythematosus in three ethnic groups: XVI. Association of hydroxychloroquine use with reduced risk of damage accrual. Arthritis Rheum 2005;52(05):1473-1480

44 Ruiz-Irastorza G, Egurbide MV, Pijoan JI, et al. Effect of antimalarials on thrombosis and survival in patients with systemic lupus erythematosus. Lupus 2006;15(09):577-583

45 Group TCHS; The Canadian Hydroxychloroquine Study Group. A randomized study of the effect of withdrawing hydroxychloroquine sulfate in systemic lupus erythematosus. N Engl J Med 1991;324(03):150-154

46 Parke AL. Antimalarial drugs, systemic lupus erythematosus and pregnancy. J Rheumatol 1988;15(04):607-610

47 Parke A. Antimalarial drugs and pregnancy. Am J Med 1988;85 (4A):30-33

48 Østensen M, Khamashta M, Lockshin M, et al. Anti-inflammatory and immunosuppressive drugs and reproduction. Arthritis Res Ther 2006;8(03):209

49 Andreoli L, Bertsias GK, Agmon-Levin N, et al. EULAR recommendations for women's health and the management of family planning, assisted reproduction, pregnancy and menopause in patients with systemic lupus erythematosus and/or antiphospholipid syndrome. 2017;76(03):476-485

50 Flint J, Panchal S, Hurrell A, et al; BSR and BHPR Standards, Guidelines and Audit Working Group. BSR and BHPR guideline on prescribing drugs in pregnancy and breastfeeding-Part I: standard and biologic disease modifying anti-rheumatic drugs and corticosteroids. Rheumatology (Oxford) 2016;55(09):1693-1697

51 Schmidt-Tanguy A, Voswinkel J, Henrion D, et al. Antithrombotic effects of hydroxychloroquine in primary antiphospholipid syndrome patients. J Thromb Haemost 2013;11(10):1927-1929

52 Hospital for Special Surgery, New York. Hydroxychloroquine for the First Thrombosis Prevention in Antiphospholipid Antibody Positive Patients. ClinicalTrials.gov identifier: NCT01784523. Available at: https://clinicaltrials.gov/ct2/show/NCT01784523. Accessed May 14, 2017

53 Mekinian A, Lazzaroni MG, Kuzenko A, et al; SNFMI and the European Forum on Antiphospholipid Antibodies. The efficacy of hydroxychloroquine for obstetrical outcome in anti-phospholipid syndrome: Data from a European multicenter retrospective study. Autoimmun Rev 2015;14(06):498-502

54 Sciascia S, Hunt BJ, Talavera-Garcia E, Lliso G, Khamashta MA Cuadrado MJ. The impact of hydroxychloroquine treatment on pregnancy outcome in women with antiphospholipid antibodies. Am J Obstet Gynecol 2016;214(02):273.e1-273.e8

55 Schreiber K, Sciascia S, Breen K, et al. Proposed trial: hypatia-a prospective randomised controlled trial of hydroxychoroquine versus placebo during pregnancy in women with antiphospholipid antibodies. J Thromb Haemost 2015;13:281

56 Pengo V, Tripodi A, Reber G, et al; Subcommittee on Lupus Anticoagulant/Antiphospholipid Antibody of the Scientific and Standardisation Committee of the International Society on Thrombosis and Haemostasis. Update of the guidelines for lupus anticoagulant detection. J Thromb Haemost 2009;7(10): $1737-1740$

57 Devreese KM, Pierangeli SS, de Laat B, Tripodi A, Atsumi T, Ortel TL; Subcommittee on Lupus Anticoagulant/Phospholipid/Dependent Antibodies. Testing for antiphospholipid antibodies with 
solid phase assays: guidance from the SSC of the ISTH. J Thromb Haemost 2014;12(05):792-795

58 MHRA. MHRA-Clinical Trial Authorisation. 2017. Available at: http://www.hra.nhs.uk/research-community/the-review-process/mhra-clinical-trial-authorisation/. Accessed May 27, 2017

59 Gardiner C, Hills J, Machin SJ, Cohen H. Diagnosis of antiphospholipid syndrome in routine clinical practice. Lupus 2013;22(01):18-25

60 Arachchillage DR, Machin SJ, Mackie IJ, Cohen H. Diagnosis and management of non-criteria obstetric antiphospholipid syndrome. Thromb Haemost 2015;113(01):13-19

61 Ruffatti $\mathrm{A}$, Olivieri $\mathrm{S}$, Tonello $\mathrm{M}$, et al. Influence of different IgG anticardiolipin antibody cut-off values on antiphospholipid syndrome classification. J Thromb Haemost 2008;6(10): 1693-1696

62 Boffa MC, Boinot C, De Carolis S, et al. Laboratory criteria of the obstetrical antiphospholipid syndrome. Data from a multicentric prospective European women cohort. Thromb Haemost 2009; 102(01):25-28

63 Cohn DM, Goddijn M, Middeldorp S, Korevaar JC, Dawood F, Farquharson RG. Recurrent miscarriage and antiphospholipid antibodies: prognosis of subsequent pregnancy. J Thromb Haemost 2010;8(10):2208-2213

64 Kaandorp SP, Goddijn M, van der Post JA, et al. Aspirin plus heparin or aspirin alone in women with recurrent miscarriage. N Engl J Med 2010;362(17):1586-1596 